\title{
Assigning Proctors to Exams with Scatter Search
}

\author{
HELENA LOURENÇO ${ }^{1}$ \\ Universitat Pompeu Fabra, Spain \\ RAFAEL MARTÍ \\ Universitat de Valencia, Spain \\ MANUEL LAGUNA \\ University of Colorado, U.S.A.
}

Abstract: In this paper we present an algorithm to assign proctors to exams. This NPhard problem is related to the generalized assignment problem with multiple objectives. The problem consists of assigning teaching assistants to proctor final exams at a university. We formulate this problem as a multiobjective integer program (IP) with a preference function and a workload-fairness function. We then consider also a weighted objective that combines both functions. We develop a scatter search procedure and compare its outcome with solutions found by solving the IP model with CPLEX 6.5. Our test problems are real instances from a University in Spain.

Keywords: multiobjective combinatorial optimization, metaheuristics, scatter search.

\footnotetext{
${ }^{1}$ Address: Departmento d'Economia i Empresa, Universitat Pompeu Fabra, R. Trias Fargas 25-27, 08005 Barcelona, Spain, helena.ramalhinho@econ.upf.es
} 


\section{INTRODUCTION}

Consider a set of teaching assistants (TAs) at a large university. Each TA has a maximum number of hours that he/she can devote to proctor final exams. This limit depends on his/her contract and teaching load. Each final exam requires a given number of TAs for proctoring. The Proctor Assignment Problem consists of assigning the TAs to the final exams. Since the TAs are graduate students, they also have final exams and therefore they cannot proctor exams during periods that conflict with their own exams.

The constraints can be summarized as follows:

- Each exam must be proctored by a specified number of TAs.

- A TA cannot exceed his/her maximum number of proctor hours.

- A TA cannot proctor more than one exam at the same time.

- A TA cannot proctor a final exam that conflicts with one of his/her own.

- A TA should proctor the exams of the courses he/she taught.

Note that the last constraint can be handled before formulating the model by simply assigning proctors to the exams of the courses they taught and adjusting the associated input data accordingly (e.g., reducing the total number of proctor hours and the exam requirements). Teaching assistants have preferences for some exams, which reflect their desire for proctoring on a given day or avoiding certain days. For example, some TAs would like to avoid proctoring an exam the day before one of their own exams. As a result of these preferences, one objective of the problem is to make assignments that maximize a function of the preferences.

Another important criterion that must be considered is to assign exams so the workload is evenly distributed among TA's. Unfair workloads are likely to generate conflicts among TA's and between TA's and the administration. Several objective functions can be formulated to measure the workload-fairness of a given assignment. One possibility is to minimize the difference between the TA with the largest workload and the one with the smallest. Alternatively, the model can seek to maximize the minimum workload associated with each TA. Since the number of available hours for each TA varies, the workload can be expressed as the ratio of assigned hours to available hours.

The Proctor Assignment Problem (PAP) can be viewed as an extension of the wellknown Generalized Assignment Problem (GAP) (see Lourenço and Serra 1998; Laguna, et al. 1995; Osman 1995; and Chu and Beasley 1997), by allowing more than one agent to be assigned to a task and introducing side constraints. The side constraints model are the aspect of the problem that are not part of the GAP, such as the need for assigning multiple proctors to an exam and for avoiding the assignment of the same TA to multiple exams that are held at the same time period. Moreover, instead of a single objective function that maximizes the total profit in the GAP, the PAP considers two objective functions to simultaneously maximize preferences and fairness. Therefore, the PAP can be formulated as multi-objective integer program. We propose a heuristic procedure based on the scatter search methodology to find solutions to the PAP.

Scatter search is an evolutionary method that has recently been shown to yield promising outcomes for solving combinatorial optimization problems. We present an 
innovative extension of the scatter search to solve multiobjective combinatorial optimization problems in general, and exemplified with the application to solve the PAP. Since the scatter-search main feature is a population-based search, we believe it will be an adequate solution technique to solve hard and large-scale multiobjective problems by finding an approximation of the set of Pareto-optimal solutions.

The procedure will be embedded in a user-friendly system to help the planning of final exams in the School of Economics and Business of the University Pompeu Fabra at Barcelona (Spain).

The paper is organized as follows: first, we present the proctor assignment problem and its mathematical programming formulation. In section 3, we describe the scatter search approach and the details of the main procedures of the scatter search developed to solve the PAP. Section 4 describes the computational experiments to evaluate the proposed heuristics, presents the computational results and performs a comparison with solutions found by the IP formulation with an utility function. Finally we conclude in section 6 with general remarks on this work and directions of future research.

\section{PROBLEM FORMULATION}

In order to simplify the problem and mimic the manual method currently practiced at the University Pompeu Fabra of Barcelona, we split an exam day into two periods (8:00 AM - 2:00 PM and 2:00 PM - 9:00 PM). Then if $d$ is the total number of exam days, $k=2 d$ is the total number of periods. The following assumptions are made:

- An exam starts and ends in the same day.

- An exam can be scheduled over one or two periods in a given day.

- TA's express their preferences for given periods.

In order to standardize the TA preferences, we translate the preferences for periods to preferences for exams. That is, if a TA expresses a strong preference for period 1 (8:00 AM - 2:00 PM in the first day of final exams), then we consider that the TA has a strong preference for all exams scheduled during this period. If an exam is scheduled over two periods (e.g., an exam that starts at noon and finishes at 3:00 PM on the first day of final exams), then we use the lower value between the preference for period 1 and 2 , as expressed by each TA.

Let $J$ be the set of $m$ exams $(j=1, \ldots, m)$ and $I$ the set of $n$ TA's $(i=1, \ldots, n)$. Then, the following notation is used to represent the relevant data in our problem:

$a_{i}=$ maximum number of available hours for TA $i$.

$b_{j}=$ number of hours associated with exam $j$.

$t_{j}=$ number of TA's required for exam $j$.

$c_{i j}=$ preference of TA $i$ for the exam $j$.

$P_{i}=$ the set of exams that overlap with any TA $i$ 's exams.

$T_{k}=$ the set of exams scheduled in period $k$.

$d=$ number of exam days. 
Also, we define the set of binary variables $x_{i j}$, such that $x_{i j}=1$ if TA $i$ is assigned to exam $j$; and $x_{i j}=0$ otherwise. Using these definitions, the PAP can be formulated as follows:

$$
\begin{array}{lll}
\text { Max } & f(x)=\sum_{i=1}^{n} \sum_{j=1}^{n} c_{i j} x_{i j} & \\
\text { Max } & g(y)=y & \\
\text { Subject to } & \sum_{j=1}^{m} b_{j} x_{i j} \leq a_{i} & i=1, \ldots, n \\
& \sum_{j=1}^{m} b_{j} x_{i j}-a_{i} y \geq 0 & i=1, \ldots, n \\
& \sum_{i=1}^{n} x_{i j}=t_{j} & \\
& \sum_{j \in T_{k}} x_{i j} \leq 1 & \\
& x_{i j}=\{0,1\} & i=1, \ldots, n ; k=1, \ldots, 2 d \\
& x_{i j}=0 & \\
& y \geq 0 & \\
& &
\end{array}
$$

Equation (1) and (2) represent the sum of the preferences and the minimum TA utilization, respectively. These objectives are to be maximized. The utilization is the fraction of the available hours that a TA is assigned to proctor exams. Constraint (3) limits the number of assigned hours to not exceed the available hours for each TA. Constraint (4) calculates the minimum TA utilization. Constraint (5) guarantees that each exam has the required number of TA's. Constraint (6) guarantees that each TA can proctor at most one exam in the same period. Constraint (7) enforces the binary restrictions on the decision variables. Constraint (8) eliminates the assignments that create a conflict with the exams that proctors have. Finally, constraint (9) enforces the nonnegativity restriction on the $y$-variable.

The usual approach to multiobjective combinatorial optimization problems is to combine the objective functions to create a single, weighted, function. For the PAP we propose the following weighted function:

$$
h(x)=f^{\prime}(x)+\alpha g(y)
$$

where $\quad f^{\prime}(x)=\frac{\sum_{i=1}^{n} \sum_{j=1}^{m} \frac{c_{i j}}{c_{\max }(j)} x_{i j}}{\sum_{j=1}^{m} t_{j}}$

and

$$
c_{\max }(j)=\max _{i}\left(1, c_{i j}\right) .
$$


Note that since the minimum preference value is zero, then both $f^{\prime}(x)$ and $g(y)$ are bounded between 0 and 1 . The value of $\alpha \geq 0$ is used to show the preference of the decision maker towards either element of the combined objective function. If $\alpha>1$, then the decision maker prefers assignments with a more uniform distribution of the workload. If $\alpha<1$, then the decision maker prefers assignments that maximize the total (normalized) preference value.

The multiobjective optimization have been vastly studied, see Steuer (1986) for a basic reference. One property that is commonly considered as necessary for any candidate solution to the multiobjective optimization problem is that the solution is not dominated.

A feasible solution $x$ is dominated by a second feasible solution $y$ in a multiobjective linear programming with $K$ objective functions if and only if $f_{k}(y) \geq f_{k}(x)$ for all the objective functions $k=1, \ldots K$, and $f_{k}(y)>f_{k}(x)$ for at least one $k$. A feasible solution is efficient if and only if there is no other feasible solution, which dominates it.

In classical literature on muldimensional optimization problem, the usual approach is to aggregate all the objectives in a utility function and solve the problem as a single objective problem, as mentioned above. However, knowing the set of efficient (Paretooptimal) solutions, or an approximation of it, gives greater freedom to the decision maker when selecting solutions, but finding this set is a complex task. If several efficient solutions are known by the user, this one can compare them even with respect to additional criteria which have not been formalized, e.g. the acceptance by management or proctors. The possibility of analyzing several scenarios of efficient solutions is very important aspect for the decision making process. For all these reasons, the possibility to obtain an approximation of the set of Pareto-optimal solutions is very important to be able to apply in real-life the program developed.

Next, we will present solution technique based on the scatter search to solve the problem, with both versions, using the utility function and also obtaining a set of efficient solutions as an approximation of the Pareto set.

\section{SCATTER SEARCH APPROACH}

Metaheuristics have been object of extensive studies and applied with success to several single-objective optimization problems, including the several generalized assignment problems, Lourenço and Serra (1998); Laguna, et al. (1995); Osman (1995); and Chu and Beasley (1997). For large list of applications and recent developments see the two volumes published as proceedings of the International Metaheuristics Conferences, Osman and Kelly (1996) and Voss et al. (1998), and see Osman and Laporte (1996) for a large list of publications. However, only recently there has been an increase interest in applying metaheuristics to multiobjective combinatorial optimization problems, see Viana and Sousa (1999), Hansen (1997) and Fonseca and Fleming (1995). The aim of multiobjective metaheuristics is to obtain a good 
approximation of the set of efficient solutions. Due the computational efficiency of these methods, we propose a multiobjective scatter search to solve the PAP.

Scatter search, from the standpoint of metaheuristic classification, may be viewed as an evolutionary (or also called population-based) algorithm that constructs solutions by combining others. It derives its foundations from strategies originally proposed for combining decision rules and constraints (in the context of integer programming). The goal of this methodology is to enable the implementation of solution procedures that can derive new solutions from combined elements in order to yield better solutions than those procedures that base their combinations only on a set of original elements. As described in tutorial articles (Glover 1998 and Laguna 1999) and other implementations based on this framework (Campos et al. 1998), the methodology includes the following basic elements:

- Generate a population $P$.

- Extract a reference set $R$.

- Combine elements of $R$ and maintain and update $R$.

Scatter search finds improved solutions by combining solutions in $R$. This set, known as the reference set, consists of solutions of high quality that are also diverse. The overall proposed procedure, based on the scatter-search elements listed above, follows:

Generate a population P: Apply the diversification generator to generate diverse solutions.

Construct the reference set $R$ : Add to $R$ the best $r_{1}$ solutions in $P$. Add also $r_{2}$ diverse solutions from $P$ to construct $R$ with $|R|=r_{1}+r_{2}$ solutions.

Maintain and update the reference ret $R$ : Apply the subset generation method (Glover 1998) to combine solutions from $R$. Update $R$ by adding solutions that improve the quality of the worst in the set.

We now describe the implementation details of the main elements of the procedure for the scatter search as adapted in the context of the exam proctor assignment.

The proposed scatter search will be innovative in at least two ways. First, there has not been a lot of work done in applying meta-heuristics to multiobjective optimization. Certainly, scatter search has not been applied in such a context. Second, in scatter search, solutions are added to $R$ because of their quality or their diversity. The number of solutions that are included because of their quality is limited by a fixed parameter $\left(r_{1}\right)$. That is, the size of $R$ is given by $r_{1}+r_{2}$, where $r_{1}$ is the number of best solutions ever found and $r_{2}$ is a number of diverse solutions. In our scatter search implementation, however, $r_{l}$ would not need to be defined, because it can be given by the size of the non-dominated set $D N$. We can then decide if we want to keep $R$ of a fixed size RefSize, by making $r_{2}=$ RefSize $-|D N|$. $R$ could also be of a variable size by fixing $r_{2}$ to a given value. 
The overall proposed scatter search method can be described as follows:

1 Read data

2 Preprocess the data: exams with a small number of students are proctor by the course's professor. TA's are assigned to the exams corresponding to the courses they taught. Exam and TA data are updated to reflect these assignments.

3 Change $=$ True

4 Generate a seed solution using the Greedy Heuristic.

5 Diversification Method.

5.1 Create the set $P$ of different solutions using the Greedy Heuristic with the greedy+frequency function.

5.2 For each solution, apply the Improvement Method and update the frequency function.

6 While (Change $==$ True) do

6.1 Construct the Reference Set $R$ considering the best no-dominated solutions in $P\left(r_{1}\right)+$ diverse solutions $\left(r_{2}\right)$.

6.2 While (Subset counter > max subset) do

6.2.1 Subset Generation Method (like in the template).

6.2.2 For each subset $X$ use the Solution Combination Method to produce a set $C(X)$ of trial solutions, using a voting system like in Campos, Laguna \& Martí (1998) but exchanging columns. The vote of each parent can be weighted by the unfeasibility created with respect to the constraints (2) and (4).

6.2.3 To each trial solution apply the Improvement Method and obtain $x^{\prime}$. If the trial solution $x^{\prime}$ is not in $R$ (Reference Set) and its a nodominated solution then:

6.2.3.1 Add $x$ ' to $R$ and remove the solutions dominated by $x$, if they exist.

6.2.3.2 Make subset counter $=0$ and repeat from 6.2. (Do not consider the subsets already analyzed).

6.3 If at least one no dominated solutions in $R$ has changed:

6.3.1 Build a new set $P$ considering the best $r_{l}$ no-dominated solutions currently in $\mathrm{R}$ and using the Diversification Generator to generate new solution; consider the frequency function for the no-dominated solutions.

6.4 Else, Change $=$ False .

Next, we will discuss in detail the main procedures of the above scatter search to solve the PAP, starting by the method to generate the initial solution.

\subsection{Diversification Generation Method}

An initial population of solutions is constructed by means of a diversification generator. The generator that we implemented is based on the notion of constructing solutions employing modified frequencies. The goal of the method is to generate goodquality diverse solutions. The generator uses the following frequency function:

$$
f_{i j}=\sum_{x \in P} x_{i j}
$$


This frequency value is used to bias the potential assignment of TA $i$ to exam $j$ during subsequent constructions of solutions, and therefore to induce diversity in the new solutions with respect to the solutions already in $P$.

The attractiveness of assigning TA to an exam is given by his/her preference. The preference of TA $i$ for exam $j\left(c_{i j}\right)$ is a value in the range [0,5]. The preference value is computed as the difference between the period in which exam $j$ is scheduled and the period of the closest exam that TA $i$ must take. Differences of more than 5 periods are adjusted back to 5. The period just before one for which a TA has an exam is assigned a preference of 0 .

We modify the value of $c_{i j}$ to reflect previous assignments of TA $i$ to exam $j$, as follows:

$$
c^{\prime}{ }_{i j}=c_{i j}-\beta\left(\frac{\max _{i, j} c_{i j}}{\max _{i, j} f_{i j}}\right) f_{i j}
$$

It should be noted that $c_{i j}^{\prime}$ is an adaptive function, since its value depends on attributes of the unassigned elements and a function of previous assignments. The value of $\beta$ is dynamically modified to encourage additional diversification. If the generator constructs the same solution more than once, the $\beta$-value is increased. In our implementation, we start with $\beta=0.4$ and increase its value by 0.1 every time the generator repeats a construction.

Figure 1 summarizes the diversification generation method. The method generates PopSize solutions using the updated ${c^{\prime}}_{i j}$ values. TA's are assigned to exams in order to maximize the modified preference values. The procedure stops when PopSize solutions are generated. 


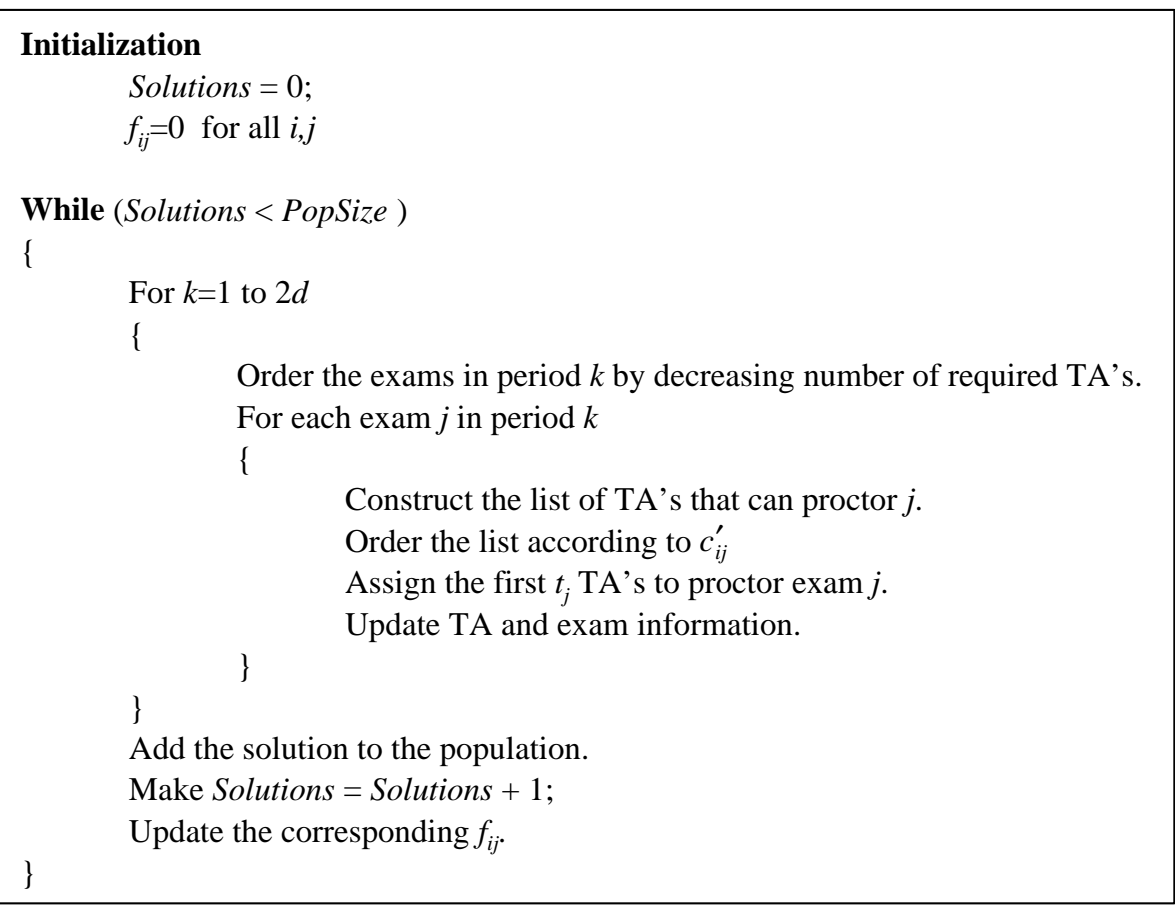

Figure 1. Diversification generator

\subsection{Improvement Method}

The improvement method pretends to improve the solutions obtained by the greedy heuristic, the diversification method and the solution combination method. We consider a simple local search method, where the neighborhood consists in exchange one TA from the list of proctors of an exam, a 2-opt neighborhood. Note that, since we pretend to have solutions where the TA have similar workload, we will start by moving exams from TAs with high workload to the ones with small.

The improvement method accepts only neighbor solutions that improve objective function $f(x)$ and do not decrease the value of $g(x)$, i.e. if exam $j$ is changed from the exams list of TA $i$ to exam list of TA $k$, then we accept the solution if $c_{i j}<c_{k j}$, and $g(y) \leq \frac{\sum_{l=1}^{m} b_{l} x_{i l}-b_{j}}{a_{i}}$, the utilization of TA $i$, after removing exam $j$. Note that the utilization of TA $k$ increases meanwhile the utilization of TA $i$ decreases. Also, if it is detected a TA with two or more exams to proctor in the same period, one of the exams is removed and assign to other TA, even if the solution does not improve. 


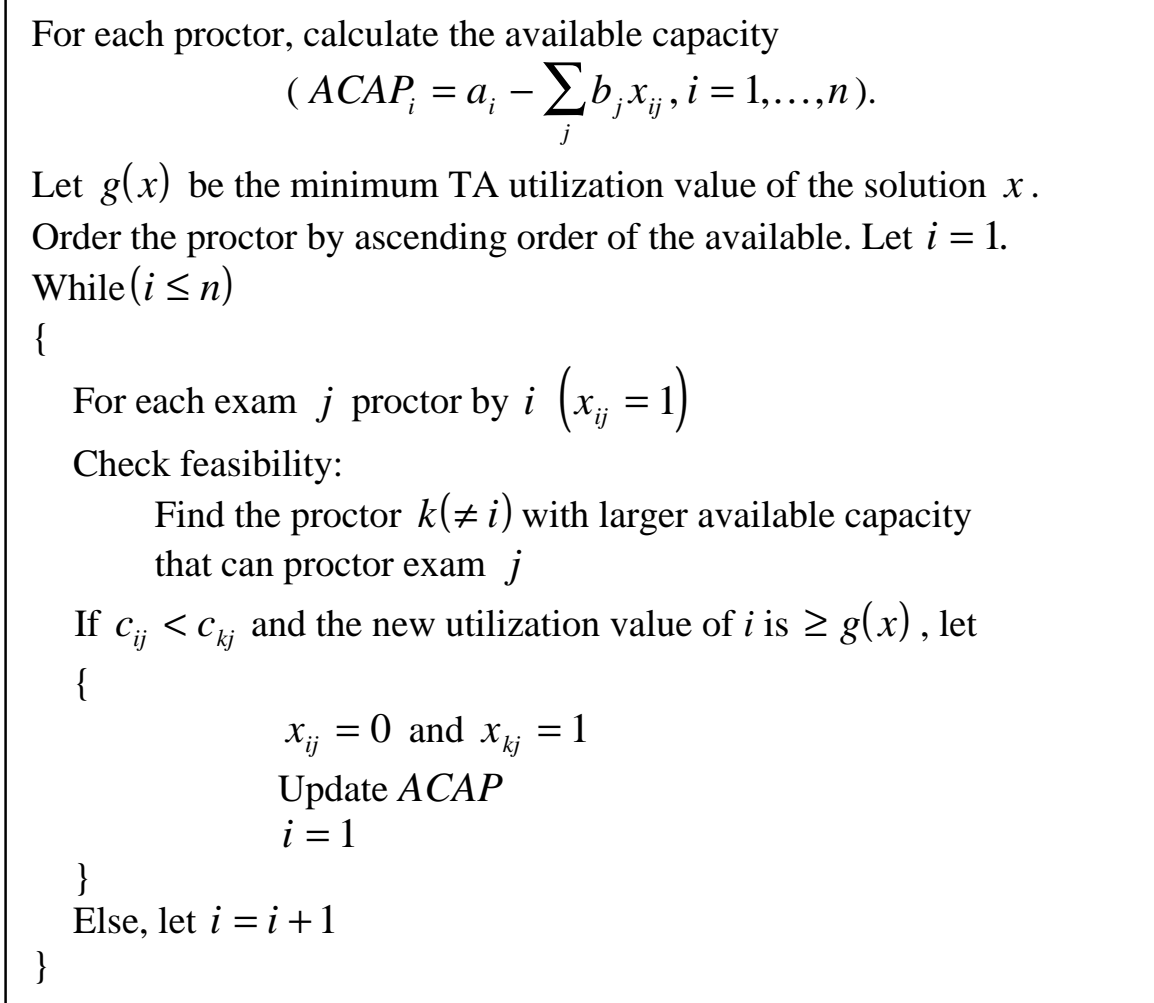

\section{Figure 2. Improvement Method}

\subsection{Updating and Maintaining the Reference Set}

The reference set $R$ is a subset of the population set $P$ that consists of high quality and diverse solutions. A distance function, $\partial\left(x^{\prime}, x^{\prime \prime}\right)$, between two solutions $x^{\prime}$ and $x^{\prime \prime}$, is used to measure the diversity of the solutions in the reference set.

$$
\partial\left(x^{\prime}, x^{\prime \prime}\right)=\sum_{i=1}^{n} \sum_{j=1}^{m}\left|x_{i j}^{\prime}-x_{i j}^{\prime \prime}\right|
$$

Note that a large distance between two solutions does not translate into a large difference between their corresponding objective function values. This is why diversity of the solutions in $R$ cannot be measure with reference to the objective function values only.

The reference set $R$ with $|R|=r$ is constructed as follows. Select the non-dominated (in the strong sense) solutions and add them to $R$. For each solution $x$ in $P$ - $R$, calculate the distance to all the elements in $R$. Let the minimum distance $\partial_{\min }(x)$ from a solution $x$ in $P-R$ to all solutions $x^{\prime}$ in $R$ be defined as:

$$
\partial_{\min }(x)=\min _{x^{\prime} \in R}\left\{\partial\left(x, x^{\prime}\right)\right\}
$$


Select the solution $x^{*}$ with the maximum distance $\partial_{\text {min }}(x)$ of all $x$ in $P-R$. Add $x^{*}$ to $R$, until $|R|=r$. In our experiments, we use $r=20$, because previous studies (Campos, et al. 1998 and 1999) have shown the merit of such choice.

Note that a solution is only inserted in the reference set if it is no dominated in the strong sense or if it is diverse from the solutions already in the set. If $x$ is strongly dominated by other solution in the reference set, do not include it this set otherwise include it. The solutions in the reference set are ordered by the value of the utility function $h(x)$. The "best solution" is the one with higher $h(x)$.

\subsection{Solution Combination Method}

Scatter search generates new solutions by combining those in the reference set. Specifically, a combination method is applied to subsets of solutions in the reference set. A newly generated solution is compared with the worst solution in $R$. The new solution replaces the solutions in $R$ are dominated by it.

The solution combination procedure seeks to generate subsets $X$ of $R$ that have useful properties, while avoiding the duplication of subsets previously generated. The approach for doing this is organized to generate four different collections of subsets of $R$, which Glover (1998) refers to as SubSetType 1, 2, 3 and 4:

SubsetType 1: all 2-element subsets.

SubsetType 2: 3-element subsets derived from the 2-element subsets by augmenting each 2-element subset to include the best solution not in this subset.

SubsetType 3: 4-element subsets derived from the 3-element subsets by augmenting each 3-element subset to include the best solution not in this subset.

SubsetType 4: the subsets consisting of the best $i$ elements, for $i=5$ to $r$.

A central consideration of this design is that $R$ itself might not be static, because it might be changing as new solutions are added to replace old ones (when these new solutions qualify to be among the current $r$ best solutions found).

The solution combination method is applied to each subset. This is based on a voting system, where each solution votes for specific assignments of TA's to exams. The resulting construction may be infeasible with respect to constraints (3) and (6), in which case, a repair mechanism is applied. The voting scheme is summarized in Figure 2.

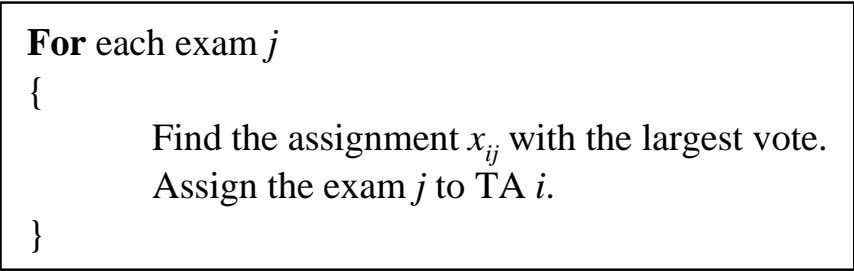

Figure 3. Voting mechanism 
The vote associated with the assignments $x_{\bullet j}$ is a measure of the merit of assigning TA's to exam $j$. The vote is therefore defined to take into account the preference of the TA's for some exams. In addition, the vote penalizes assignments that result in violations of one or more of the problem constraints.

The process consists of $m$ steps (where $m$ is the number of exams). At each step $j$, a solution $x$ votes for its column $x_{\bullet j}$, that consists of all the TA assignments associated with exam $\mathrm{j}$. The vote of solution $x$ at step $j$ is calculated as follows:

$$
V_{j}(x)=\sum_{i=1}^{n}\left(\begin{array}{l}
\left.c_{i j} x_{i j}-\varphi\left(\max \left(0, \sum_{l=1}^{j-1} b_{l} y_{i l}+b_{j} x_{i j}-a_{i}\right)\right)\right) \\
-\gamma\left(\max \left(0, \sum_{k: j \in T_{k}}\left(\sum_{l \in T_{k}, l \leq j-1} y_{i l}+x_{i j}-1\right)\right)\right)
\end{array}\right)
$$

The first term of the vote calculation adds the preferences of the TA's assigned to exam $j$ in solution $x$. The second term calculates the number of hours assigned to each TA in the solution that is being constructed (represented by the $y$-variable). Then, it adds the number of hours for the current exam $j$ and then subtracts the available hours for the TA. If the hours already assigned plus the hours related to the current exam exceed the available number of hours, then the excess hours are multiplied by a penalty factor $\varphi$. In other words, the second term in equation (17) penalizes violations of constraint (3). In a similar way, the third term of equation (1) penalizes the violation of constraint (6), by multiplying the number of times a TA is assigned to proctor different exams in the same time period by the constant $\gamma$.

When the combination method results in a solution that violates either constraint (3) or constraint (6), the procedure in Figure 3 is executed in an attempt to repair the newly generated solution.

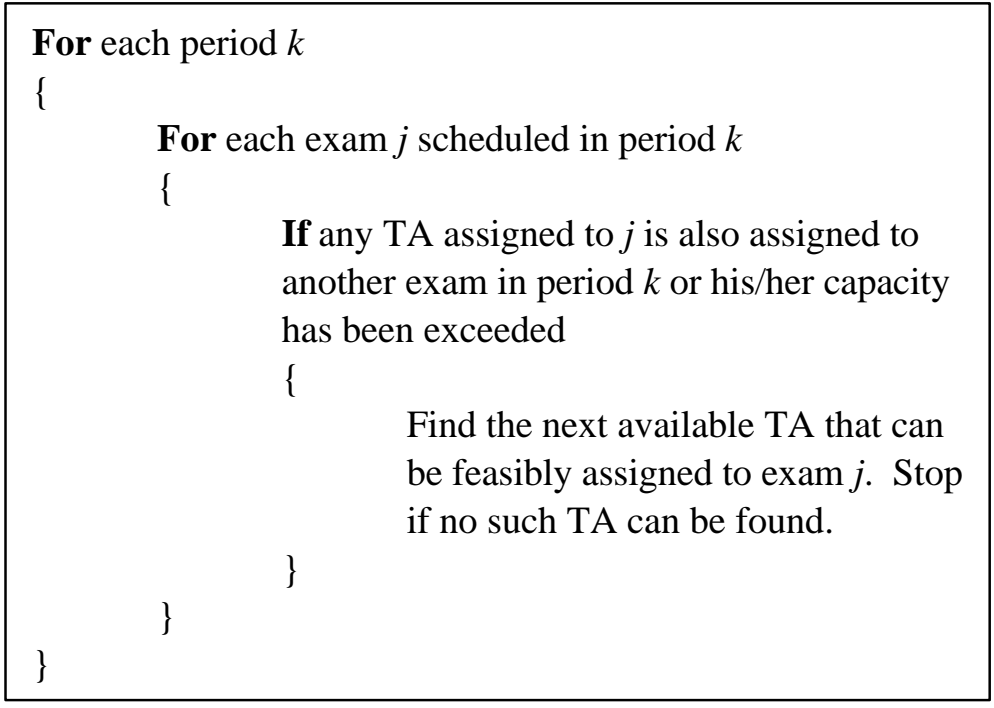

Figure 4. Solution repair procedure

Although the procedure in Figure 2. may fail to repair a solution, our experience has been that the method seldom fails. 


\subsection{The restart}

We restart the process with the set of non-dominated solutions in the reference set. Note that a solution is only inserted in the reference set if it is no dominated in the strong sense. A feasible solution $x$ is strongly dominated by a second feasible solution $y$ if and only if $f_{k}(y)>f_{k}(x)$ for $k=1, \ldots K$. A solution is dominated in a strong sense if both objective functions are worst than other solution in the reference set. All solutions in the reference set are included in the population, and the frequency function is actualized. Then, we generate the remaining of the population using the diversification generator method. If during one scatter search iteration a new solution is inserted in the reference set, then we repeat the process. Therefore the method terminates if no solution is inserted in the reference set.

\section{COMPUTATIONAL EXPERIMENTS}

The data used for these experiments correspond to real instances of the proctor assignment problem at the Universitat Pompeu Fabra in Barcelona (Spain). Presently, proctors are manually assigned to exams, following simple rules that enforce the constraints in the problem. We compare our results with assignments that were generated manually and also with assignments found solving the mixed-integer programming formulation with Cplex 6.5 (some of which are optimal).

Our test set consists of 11 problems. The results are summarized in Table 1. The first three columns in this table show the problem number, the number of TA's and the number of exams, respectively. The table than shows the objective function value corresponding to the scatter search solution and the solution found with Cplex. The asterisk in the Cplex column indicates that the solution was confirmed to be optimal.

Table 1 shows that scatter search solutions are inferior to those found by Cplex in the set of problems used for testing when the utility function is used. However, an advantage of scatter search is that the reference set contains a number of high-quality solutions from which the decision-maker could choose the one to implement. The maximum standard deviation of the utility function value for solutions in the final reference set was 0.000407 for all problem instances. This indicates that practically all of the solutions in the final reference set have the same quality with respect to the objective function value. Since the utility function is a mathematical representation of some subjective measure of performance associated with a given assignment, the ability to choose among solutions that have similar objective function values is an important feature of a solution procedure to be embedded in a decision support system designed for this managerial situation. 
Table 1. Summary of results

\begin{tabular}{rrrrc}
\hline Problem & \multicolumn{1}{l}{ TA's } & Exams & $\begin{array}{c}\text { Scatter } \\
\text { Search }\end{array}$ & $\begin{array}{c}\text { Cplex } \\
\text { (* optimal) }\end{array}$ \\
\hline 1 & 23 & 21 & 0.764 & 0.953 \\
2 & 59 & 52 & 1.000 & 1.393 \\
3 & 59 & 44 & 1.000 & 1.165 \\
4 & 25 & 21 & 0.754 & 0.815 \\
5 & 46 & 42 & 0.952 & 1.268 \\
6 & 92 & 84 & 1.000 & 1.308 \\
7 & 139 & 125 & 1.000 & $1.371^{*}$ \\
8 & 139 & 125 & 1.000 & $1.000^{*}$ \\
9 & 139 & 125 & 1.000 & $1.000^{*}$ \\
10 & 166 & 182 & 0.999 & $1.000^{*}$ \\
11 & 180 & 210 & 0.116 & $1.000^{*}$ \\
\hline
\end{tabular}

The scatter search procedure was coded in $\mathrm{C}$ and run on a Pentium II computer at $350 \mathrm{MHz}$. The computational times are very reasonable, with an average of 102.3 seconds and a maximum of 208.2 seconds. Cplex 6.5 was also run on a Pentium II machine at $350 \mathrm{MHz}$. The depth-first search strategy was used with a time limit of one hour.

Table 2. Results of the different versions of the scatter search.

\begin{tabular}{|c|c|c|c|c|c|c|c|c|c|}
\hline & OPT. & \multicolumn{2}{|l|}{ Utility function } & \multicolumn{2}{|c|}{$\begin{array}{l}\text { Utility function } \\
\text { Improvem. }\end{array}$} & \multicolumn{2}{|c|}{$\begin{array}{c}\text { Multiobjective } \\
\text { Scatter Search }\end{array}$} & \multicolumn{2}{c|}{$\begin{array}{c}\text { Multiobjective } \\
\text { SS+ Restart }\end{array}$} \\
\hline & & & & & & & & \multicolumn{2}{|c|}{ alpha=10 } \\
\hline Examples & & $\mathbf{h}(\mathbf{x})$ & time & $\mathbf{h}(\mathbf{x})$ & time & $\mathbf{h}(\mathbf{x})$ & time & $\mathbf{h}(\mathbf{x})$ & time \\
\hline $\mathbf{1}$ & $\mathbf{0 . 9 5 3}$ & 0.764 & 3.60 & 0.775 & 4.44 & 0.784 & 4.72 & 0.896 & 9.39 \\
\hline $\mathbf{2}$ & $\mathbf{1 . 3 9 3}$ & 1.000 & 15.68 & 1.000 & 27.08 & 1.000 & 28.01 & 1.000 & 9.01 \\
\hline $\mathbf{3}$ & $\mathbf{1 . 1 6 5}$ & 1.000 & 12.56 & 1.000 & 11.81 & 1.000 & 12.63 & 1.000 & 8.73 \\
\hline $\mathbf{4}$ & $\mathbf{0 . 8 1 5}$ & 0.754 & 3.17 & 0.755 & 0.61 & 0.755 & 6.04 & 0.755 & 7.42 \\
\hline $\mathbf{5}$ & $\mathbf{1 . 2 6 8}$ & 0.950 & 13.96 & 0.951 & 14.83 & 0.951 & 17.91 & 0.963 & 20.38 \\
\hline $\mathbf{6}$ & $\mathbf{1 . 3 0 8}$ & 1.000 & 76.88 & 1.000 & 47.67 & 1.000 & 49.22 & 1.000 & 34.93 \\
\hline $\mathbf{7}$ & $\mathbf{1 . 3 7 1}$ & 1.000 & 238.10 & 1.000 & 129.62 & 1.000 & 128.96 & 1.000 & 116.49 \\
\hline $\mathbf{9}$ & $\mathbf{1}$ & 1.000 & 140.35 & 1.000 & 129.73 & 1.000 & 131.76 & 1.000 & 140.44 \\
\hline $\mathbf{1 0}$ & $\mathbf{1}$ & 1.000 & 137.59 & 1.000 & 129.74 & 1.000 & 135.50 & 1.000 & 154.67 \\
\hline $\mathbf{1 1}$ & $\mathbf{1}$ & 0.999 & 201.37 & 0.998 & 520.64 & 0.999 & 277.76 & 0.999 & 278.74 \\
\hline $\mathbf{1 2}$ & $\mathbf{1}$ & 0.116 & 339.88 & 1.000 & 493.95 & 1.000 & 378.27 & 1.000 & 438.19 \\
\hline
\end{tabular}

In table two, we present the results of the different tested versions of the scatter search, using the utility function and the multiobjective version of the method. If we consider the utility function, we can observe that the multiobjective version with restart gave the best results overall the different proposed versions. However, the user may prefer the multiobjective scatter search versions since able him or her to analyze several very good solutions and make the final decision. 


\section{CONCLUSIONS}

One of the most interesting features of the proctor assignment problem is its multiobjective nature. In the initial development, we have formulated the problem as a mixed-integer program with a single objective, the utility function, and implemented a scatter search solution procedure. Afterwards, a multiobjective scatter search has developed, which include some innovative aspects that can also be applied to other multiobjective combinatorial optimization problems. The main features is by constructing and updating the reference set using the set of non-dominated solutions. Also, the cardinality of the reference set varies with the set of non-dominated solutions. The implementation of a procedure that uses these definitions has given an improvement on the results, but more important at all, it will permit to develop a program better fitted to be used in real decision processing making.

The multiobjective scatter search proposed in this work can be extended to other multiobjective combinatorial optimization, and we would like to evaluate the performance of such approach to well-known combinatorial optimization with several objective functions. Also, as part of future work, we would like to finish the decision support system to proctor assignment and used in several universities with similar problems.

\section{REFERENCES}

Awad, R. and J. Chinneck (1998) "Proctor Assignment at Carleton University," Interfaces, vol. 28, no. 2, pp. 58-71.

Campos, V., F. Glover, M. Laguna and R. Martí (1999) “An Experimental Evaluation of a Scatter Search for the Linear Ordering Problem," University of Colorado at Boulder.

Campos, V., M. Laguna and R. Martí (1998) "Scatter Search for the Linear Ordering Problem," to appear in New Methods in Optimisation, D. Corne, M. Dorigo and F. Glover (Eds.), McGraw-Hill.

Chu, P. C. and J. E. Beasley (1997) "A Genetic Algorithm for the Generalized Assignment Problem," Computers and Operations Research, vol. 24, pp. 17-23.

Fonseca C.M. and P.J. Fleming (1995) “An overview of evolutionary algorithms in multiobjective optimization”, Evolutionary Computation, 3:1-16.

Glover, F. (1998) "A Template for Scatter Search and Path Relinking," in Artificial Evolution, Lecture Notes in Computer Science 1363, J.-K. Hao, E. Lutton, E. Ronald, M. Schoenauer and D. Snyers (Eds.), Springer-Verlag, pp. 13-54.

M.P. Hansen (1997) "Tabu search for multiobjective optimization: Mots", Proceedings of the $13^{\text {th }}$ International Conference on Multiple Criteria Decision Making, University of Cape Town, pages 574-586. 
Laguna, M., J. P. Kelly, J. L. González Velarde and F. Glover (1995) “Tabu Search for the Multilevel Generalized Assignment Problem," European Journal of Operational Research, vol. 82, pp. 176-189.

Laguna, M. (1999) "Scatter Search", to appear in Handbook of Applied Optimization, P. M. Pardalos and M. G. C. Resende (Eds.), Oxford Academic Press.

Lourenco HR and Serra D (1998), "Adaptive approach heuristics for the generalized assignment problem”, DEE-UPF, Economic Working paper Series No. 288, May.

Osman, I. H. (1995) "Heuristics for the Generalized Assignment Problem: Simulated Annealing and Tabu Search Approaches," OR Spektrum, vol. 17, pp. 211225.

Osman I.H. and J.P. Kelly (1996) "Meta-Heuristics: Theory and Applications", Kluwer Academic Publishers.

Osman I.H. \& G. Laporte (1996) "Metaheuristics: a bibliography", in Annals of Operational Research: Metaheuristics in Combinatorial Optimization, G. Laporte \& I.H. Osman (eds), Baltzer Science Publications, vol. 63, pp. 513-628.

Steuer R.E. (1986) "Multiple criteria optimization: Theory, computation, and application”, John Wiley.

Viana A. and J. Pinho de Sousa (1999) "Some notes on Multiobjective Metaheuristics - a tutorial example", submitted for publication at the European Journal.

Voss, S. Martello, I.H. Osman, C. Roucairol (1998) "Metaheuristics: advances and trends in local search paradigms for optimization", Kluwer Academic Publishers. 